\title{
Iatrogenic subclavian artery puncture repair with Angio-Seal deployment and balloon occlusion
}

\author{
Aleksandra Gąsecka, Aleksandra Idzik, Anna Burban, Arkadiusz Pietrasik \\ $1^{\text {st }}$ Chair and Department of Cardiology, Medical University of Warsaw, Warsaw, Poland
}

Adv Interv Cardiol 2021; 17, 3 (65): 330-331

DOI: https://doi.org/10.5114/aic.2021.109387

A central venous catheter (CVC) is used during highrisk operations to prevent hypovolemic shock. Whereas arterial puncture during CVC insertion is quite frequent (2.9\%), arterial cannulation is a less common complication $(0.2 \%)$ [1]. We describe the successful removal of a CVC, inadvertently placed in the subclavian artery, with a collagen-based Angio-Seal closure device (St. Jude Medical, US).

A 90-year-old woman was admitted for emergency cardiac surgery due to type A (DeBakey II) aortic dissection. Following valve sparing ascending aorta replacement using a $26 \mathrm{~mm}$ Dacron graft (InterVascular, France) under peripheral hemodynamic monitoring (left radial artery), the decision was made to insert a CVC into the subclavian vein for catecholamine support and blood transfusion. The right subclavian vessel was cannulated with a straight needle using an ultrasound (US)-guided approach. A J-wire was passed through the needle down into the presumed subclavian vein and a 7-French CVC was advanced over the J-wire. Immediately after opening of the CVC channel, the pulsatile flow of red blood was visible, indicating subclavian artery cannulation. Chest $\mathrm{X}$-ray confirmed the CVC malposition (Figure $1 \mathrm{~A}$ ). To prevent re-thoracotomy, the Heart Team opted for endovascular closure of the arterial puncture site.

Dual arterial access was achieved: (i) via the right radial artery to enable balloon occlusion during CVC removal and (ii) via the right femoral artery to perform control angiography. Angiography confirmed the arterial placement of the CVC, with the entry site in the proximal part of the subclavian artery and the tip in the vascular prosthesis (Figure $1 \mathrm{~B}$ ). A peripheral semicompliant angioplasty balloon $(8 \times 40 \mathrm{~mm}$, Mustang, Boston Scientific, US) was advanced over a 0.035" guidewire and positioned in the subclavian artery at the level of the CVC entry site, avoiding the origin site of the vertebral or common carotid artery. As the balloon was inflated, the CVC was withdrawn over the Angio-Seal wire (Figure $1 \mathrm{C}$ ). No bleeding at the skin access site was visible following vascular suture creation. The balloon was deflated and control angiography showed no contrast extravasation (Figure $1 \mathrm{D}$ ), confirming successful artery closure. A new CVC was placed via the left femoral vein. Following 6 weeks of in-hospital rehabilitation, the patient was discharged home.

latrogenic CVC insertion into the subclavian artery is a potentially life-threatening complication. In our patient, it also posed the risk of infection due to the location next to the aortic prosthesis. The US-guided approach reduces the risk of arterial puncture and hematoma formation, but has no effect on other complications [2]. Percutaneous management of inadvertent artery cannulation includes the use of percutaneous closure devices, prolonged balloon occlusion (5-50 min) and stent-graft placement [3, 4]. Angio-Seal is licensed for use in femoral arterial puncture sites only. However, the off-label use of Angio-Seal was shown to be efficient and safe in the case of inadvertent subclavian artery puncture as well [3]. Since effective hemostasis was crucial in our patient, we combined the use of the Angio-Seal device with transient balloon rescue occlusion to maximize the procedural safety. The combination of both techniques provided safe and effective closure of the subclavian artery puncture site.

\section{Conflict of interest}

The authors declare no conflict of interest. 

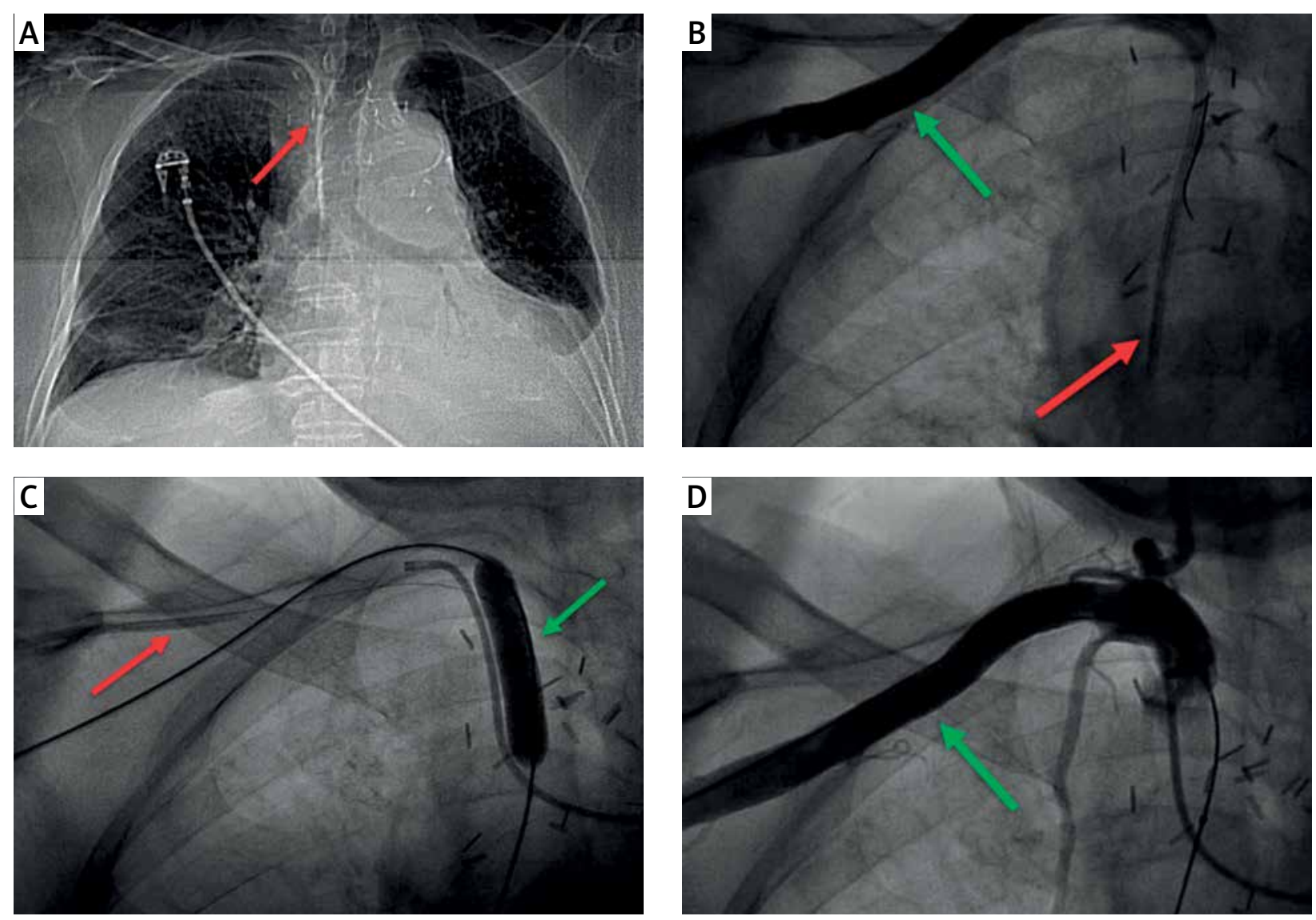

Figure 1. A - Chest X-ray showing central venous catheter (CVC) inadvertently inserted into the right subclavian artery (red arrow). B - Angiography confirmed the right subclavian arteriography cannulation (green arrow), with the CVC tip in the vascular prosthesis of the ascending aorta (red arrow). C - CVC (red arrow) is being removed over a guidewire of the Angio-Seal closure device (St. Jude Medical, Minnesota, US). The artery insertion site is secured with a balloon tamponade (green arrow). D - Control angiography of the right subclavian artery after CVC removal, showing no sign of contrast extravasation or bleeding

\section{References}

1. Gibson F, Bodenham A. Misplaced central venous catheters: applied anatomy and practical management. Br J Anaesth 2013; 110: 333-46.

2. Brass P, Hellmich M, Kolodziej L, et al. Ultrasound guidance versus anatomical landmarks for subclavian or femoral vein catheterization. Cochrane Database Syst Rev 2015; 1: CD011447.

3. Cohen JE, Gomori JM, Anner H, Itshayek E. Inadvertent subclavian artery cannulation treated by percutaneous closure. J Clin Neurosci 2014; 21: 1973-5.

4. Dunham GM, Vaidya SS. Balloon tamponade repair after inadvertent subclavian artery catheterization. J Vasc Access 2015; 16: 152-7. 